\title{
Kinetics of Glass Transition and Crystallization in Carbon Nanotube Reinforced Mg-Cu-Gd Bulk Metallic Glass
}

\author{
Wang Yinchun (王银春) ${ }^{1}$, Wang Yuren (王育人) ${ }^{1 *}$, Wei Bingchen (魏炳忱) ${ }^{1}$, Li Weihuo (李维火) ${ }^{1}$, \\ Sun Yufeng (孙玉峰) ${ }^{2}$ \\ (1. Institute of Mechanics, Chinese Academy of Sciences, Beijing 100080, China; 2. Research Center for \\ Materials, Department of Materials Science and Engineering, Zhengzhou University, Zhengzhou 450002, China)
}

Received 6 April 2005; revised 25 June 2005

\begin{abstract}
Mg}_{65} \mathrm{Cu}_{25} \mathrm{Gd}_{10}$ bulk metallic glass and its carbon nanotube reinforced composite were prepared. Differential scanning calorimeter (DSC) was used to investigate the kinetics of glass transition and crystallization processes. The influence of CNTs addition to the glass matrix on the glass transition and crystallization kinetics was studied. It is shown that the kinetic effect on glass transition and crystallization are preserved for both the monothetic glass and its glass composite. Adding CNTs in to the glass matrix reduces the influence of the heating rate on the crystallization process. In addition, the CNTs increase the energetic barrier for the glass transition. This results in the decrease of GFA. The mechanism of the GFA decrease was also discussed.
\end{abstract}

Key words: metallic glass; carbon nanotube; glass transition; crystallization; kinetic effect; composite glass; Gadolinium; rare earths

CLC number: TG139 Document code: A Article ID: $1002-0721(2006) 03-0327-05$

Bulk metallic glasses (BMGs) have unique properties such as high strength, toughness and good corrosion resistance ${ }^{[1]}$. Among the BMGs developed so far, Mg-TM-RE ( TM, transition metal; RE, rare earth metal) alloy system has gotten more and more attention for its excellent glass forming ability and high specific strength $^{[2 \sim 5]}$. Recently, Kim ${ }^{[4]}$ succeeded in manufacturing bulk $\mathrm{Mg}_{65} \mathrm{Cu}_{25} \mathrm{Gd}_{10}$ glassy alloy with enhanced glass forming ability. $\mathrm{Mg}$ based $\mathrm{BMG}$ are of interest as matrix of composite materials because of their low melting points and large undercooled liquid regions. Carbon nanotube, as the "ultimate" carbon fiber, is well known for its unique mechanical properties such as high modulus and toughness, so it can be intro- duced into other materials as a promising reinforcement. In recent years, many efforts have been dedicated to the study of CNTs reinforced polymer and metallic composites ${ }^{[6 \sim 10]}$. Wang et al. ${ }^{[11]}$ studied the influences of CNTs on the ultrasonic properties of $\mathrm{Zr}_{52.5}$ $\mathrm{Cu}_{17.9} \mathrm{Ni}_{14.6} \mathrm{Al}_{10} \mathrm{Ti}_{5}$ BMG. However, the kinetic behavior of glass transition and crystallization of CNTs reinforced BMG composite was not concerned in their study. This paper is focused on the study of the glass transition and crystallization behavior of $\mathrm{Mg}_{65} \mathrm{Cu}_{25} \mathrm{Gd}_{10}$ bulk metallic glass and its CNTs reinforced composite.

\section{Experimental}

Pure $\mathrm{Cu}$ and Gd elements (purity $\sim 99.9 \%$ ) were

\footnotetext{
* Corresponding author (E-mail: wangyr @imech.ac.cn)

Foundation item: Project supported by Knowledge Innovation Program of Chinese Academy of Sciences (KJCX2-SW-L05), and the National Natural Science Foundation of China (50101012)

Biography: Wang Yinchun (1983-), Male, Master
}

Copyright (C2006, by Editorial Committee of Journal of the Chinese Rare Earths Society. Published by Elsevier B.V. All rights reserved. 
arc-melted in Ti-gettered argon atmosphere to produce intermediate $\mathrm{Cu}-\mathrm{Gd}$ alloys. Master alloys were prepared by induction-melting the intermediate $\mathrm{Cu}-\mathrm{Gd}$ alloy together with $\mathrm{Mg}$ (purity $99.99 \%$ ) in a quartz tube under vacuum. When the master alloys were homogeneously melted, they were cast into a copper mold to get a BMG cylinder with a length of $50 \mathrm{~mm}$ and diameter of $3 \mathrm{~mm}$. In order to fabricate CNTs reinforced composites, the pre-alloyed $\mathrm{Mg}-\mathrm{Cu}-\mathrm{Gd}$ ingots were ground mechanically into fine powder. The CNT powder was cleaned in acetone and dehydrated at $473 \mathrm{~K}$ before mixing. The ingot powder was mixed homogenously with CNTs powder (3\% (volume fraction)), and then the mixed powder was compressed into cylinders in steel dies (diameter $=$ $5 \mathrm{~mm}$ ). The cylinders were melted in a quartz tube rapidly by induction under a pure argon atmosphere and then cast into a copper mold to produce composite rods with a diameter of $3 \mathrm{~mm}$.

The phase and atomic structure of the samples were investigated by $\mathrm{X}$-ray diffraction (XRD) using a MAC M03 XHF diffractometer. The microstructure of the samples was analyzed using scanning electron microscopy (HITACHI S-570). The thermal stability was examined by differential scanning calorimeter (Netzsch DSC 404) at various heating rates of $5,10,20$, and $40 \mathrm{~K} \cdot \mathrm{min}^{-1}$ under purified argon. At each heating rate, the calorimeter was adjusted using the purified In and $\mathrm{Zn}$. The onset temperature of glass transition

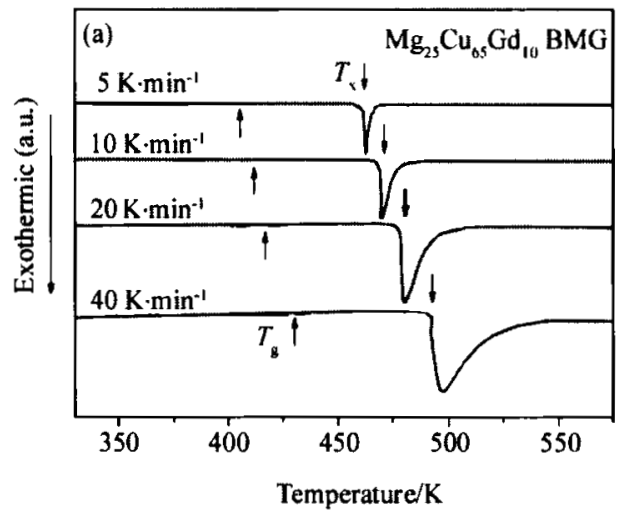

$T_{\mathrm{B}}$ onset , onset crystallization temperature $T_{x}$, and crystalline peak temperature $T_{\mathrm{p}}$ were determined by DSC traces with an accuracy of $\pm 1 \mathrm{~K}$.

\section{Results and Discussion}

\subsection{Experimental results}

Figs. 1 (a), (b) show DSC curves obtained from BMG and CNTs composite at different heating rates. Fig. 1(a) illustrates that $T_{\mathrm{g}}, T_{\mathrm{x}}$ and $T_{\mathrm{p}}$ of BMC move to higher temperature with increasing of heating rate. Not only crystallization but also glass transition shows a strong dependence on the heating rate during continuous heating, indicating that both the glass transition and crystallization processes have significant kinetic effects. This phenomenon is also reported for $\mathrm{Zr}$-based BMGs by Wang et al ${ }^{[12]}$. Fig. 1(b) shows that similar trend exists in CNTs composite, which implies that CNTs addition cannot change the kinetic effects of glass transition and crystallization. Values of $T_{\mathrm{g}}, T_{\mathrm{x}}$, $T_{\mathrm{p}}$ and SLR, $\Delta T\left(=T_{\mathrm{x}}-T_{\mathrm{g}}\right)$ for both monothetic BMG and CNTs composite at a heating rate of $20 \mathrm{~K} \cdot \mathrm{min}^{-1}$ are listed in Table 1 . With CNTs addition, $T_{\mathrm{x}}, T_{\mathrm{p}}$ and $\Delta T$ of BMG decrease about $20 \mathrm{~K}$, indicating that GFA and thermal stability of BMG are reduced, but the dependence of glass transition and crystallization behavior on heating rate is retained.

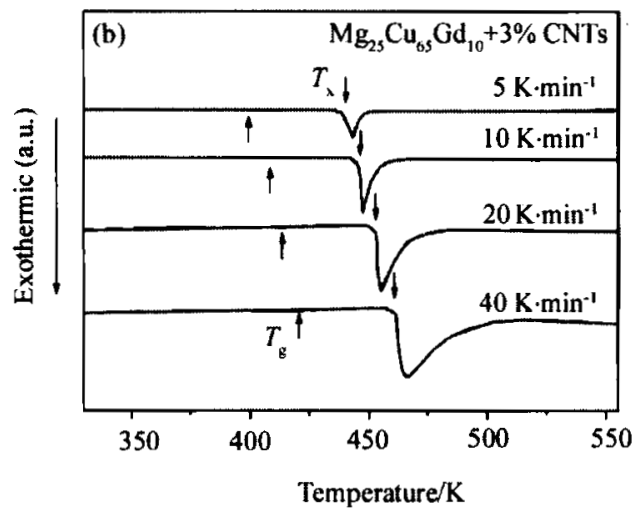

Fig. 1 DSC curves of $\mathrm{Mg}$ - $\mathrm{Cu}-\mathrm{Gd}$ BMG and its CNTs composite at different heating rates

(a) $\mathrm{Mg}_{65} \mathrm{Cu}_{25} \mathrm{Cd}_{10}$ bulk metallic glass; (b) CNTs-BMG composite

Table $1 T_{\mathrm{g}}, T_{\mathrm{x}}, T_{\mathrm{p}}$ and $\Delta T$ of $\mathrm{Mg}-\mathrm{Cu}-\mathrm{Gd} \mathrm{BMG}$ and its CNTs composite at different heating rates

\begin{tabular}{llllll}
\hline Samples & Heating rates $/\left(\mathrm{K} \cdot \min ^{-1}\right)$ & $T_{\mathrm{g}} / \mathrm{K}$ & $T_{\mathrm{x}} / \mathrm{K}$ & $T_{\mathrm{p}} / \mathrm{K}$ & $\Delta T\left(T_{\mathrm{x}}-T_{\mathrm{g}}\right) / \mathrm{K}$ \\
\hline $\mathrm{Mg}_{65} \mathrm{Cu}_{25} \mathrm{Gd}_{10} \mathrm{BMG}$ & 5 & 405.3 & 461.6 & 462.4 & 56.3 \\
& 10 & 409 & 469.1 & 470.6 & 60.1 \\
& 20 & 415 & 478.3 & 480.6 & 63.3 \\
& 40 & 425.8 & 491.6 & 497.2 & 65.8 \\
$\mathrm{CNTs}-\mathrm{BMG}$ composite & 5 & 400.4 & 439.6 & 443.4 & 39.2 \\
& 10 & 408.2 & 446.4 & 447.6 & 38.2 \\
& 20 & 413.3 & 453.2 & 455.4 & 39.9 \\
\hline
\end{tabular}




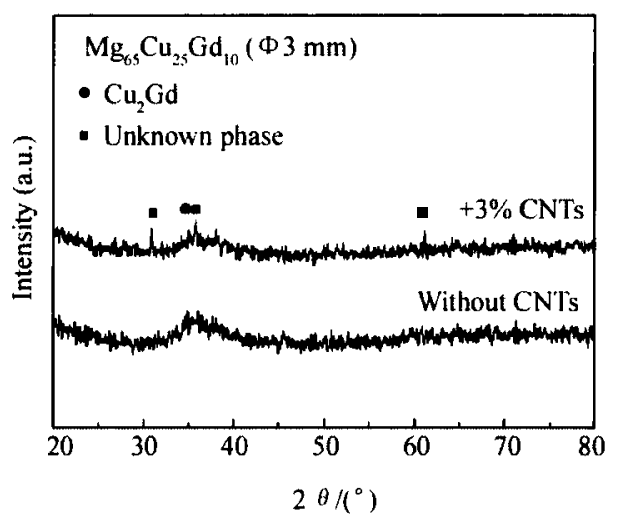

Fig.2 XRD patterns of Mg-Cu-Gd BMG and its CNTs composite

X-ray diffraction patterns of undoped BMG and $3 \%$ CNT-reinforced BMG composite are shown in Fig.2. The curve of the composite exhibits a superimposition of broad maximum from amorphous phase and several sharp peaks indicating the existence of crystalline phase. The crystalline peaks are identified as $\mathrm{Cu}_{2} \mathrm{Gd}$ phase and an unknown phase. SEM images of the BMG and its composite are shown in Fig. 3. The precipitated phase can be well identified.
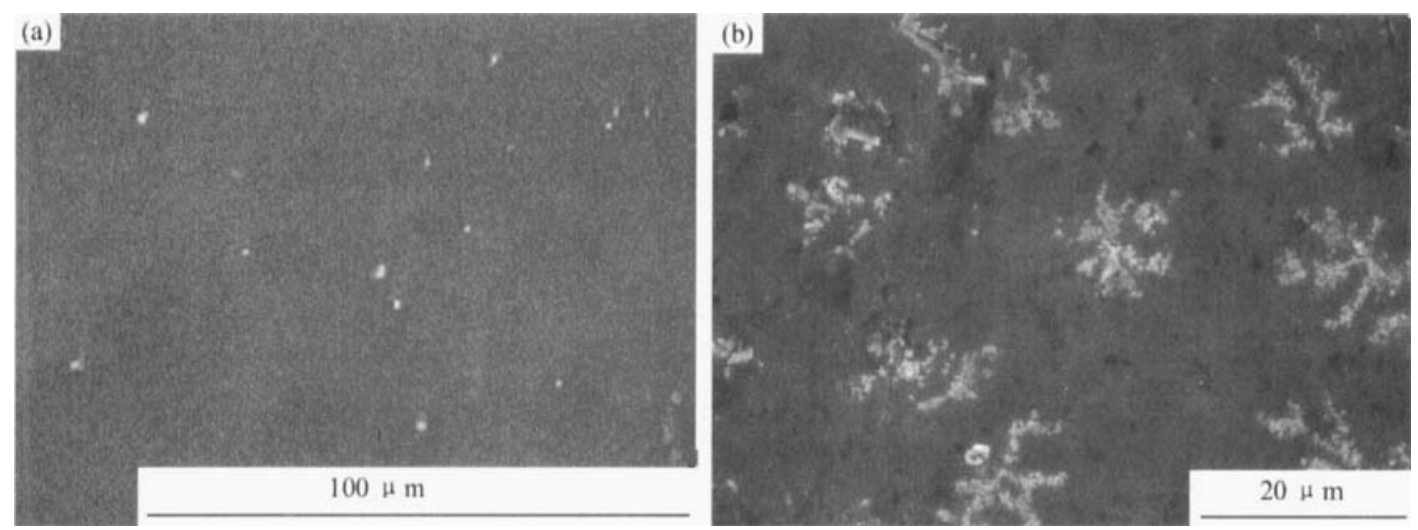

Fig. 3 SEM images taken from cross-sectional surface of as-cast rods

(a) Mg-Cu-Gd BMG; (b) CNTs-BMG composite
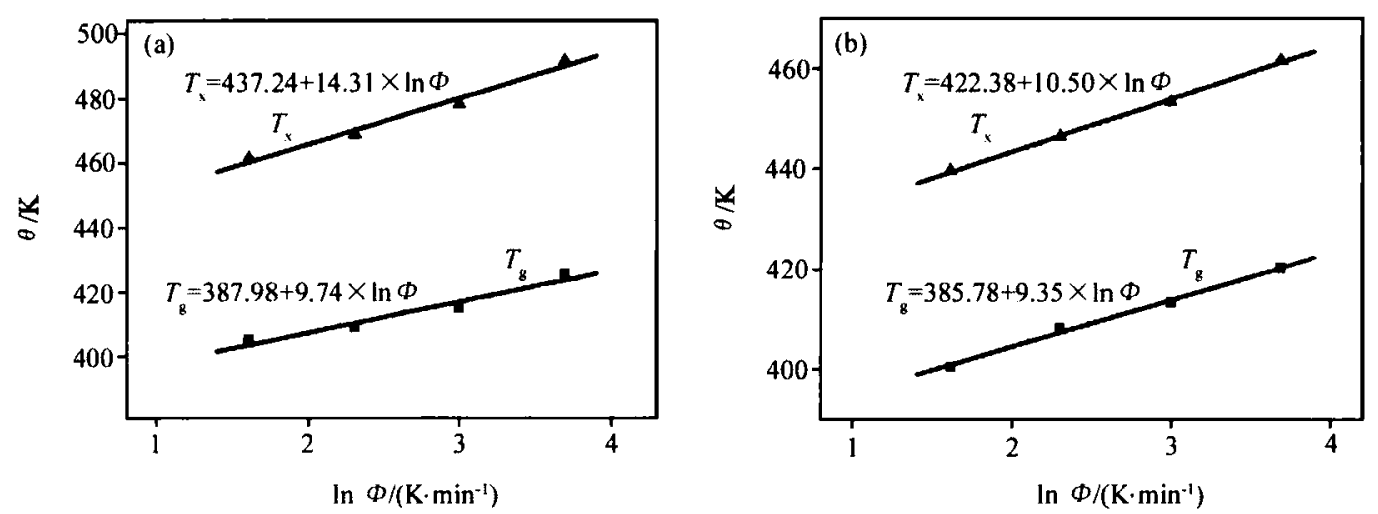

Fig.4 Plots of $T_{\mathrm{B}}, T_{\mathrm{x}}$ vs. $\ln \Phi$ for Mg-Cu-Gd BMG (a) and CNTs-BMG composite (b) 
Cu-Gd BMG and its CNTs composite, respectively, under a heating rate of $5 \mathrm{~K} \cdot \mathrm{min}^{-1}$. It indicates the occurrence of second-order phase transition from glass state to supercooled liquid state, which has been discussed in Ref. [14].

\subsection{GFA and crystallization kinetics}

During crystallization process, atoms participating in crystallization reaction should acquire additional energy to form nuclei of crystallization. The activation energy can be interpreted as the additional energy acquired by an atom before it becomes a part of the nuclei. Kissinger method is widely used in calculating the activation energy in $\mathrm{BMG}^{[15]}$.

Kissinger equation can be described as follows:

$\ln \frac{\theta^{2}}{\Phi}=\frac{E}{k_{\mathrm{B}} \theta}+\ln \frac{E}{k_{\mathrm{B}} v_{0}}$

where $\theta$ stands for $T_{\mathrm{g}}$ or $T_{\mathrm{x}}, \Phi$ is the heating rate represents Boltzmann constant. $k_{\mathrm{B}}$ is the value of crystallization constant. The value of crystallization activation energy $E$ can be calculated from the slope. Fig. 5 shows the Kissinger plots of glass transition and crystallization for monothetic BMG and its CNTs composite. There exists a strong linear relation between $\ln \left(\theta^{2} / \Phi\right)$ and $1 / T$ in spite of CNTs addition. The apparent activation energy of glass transition increases af-

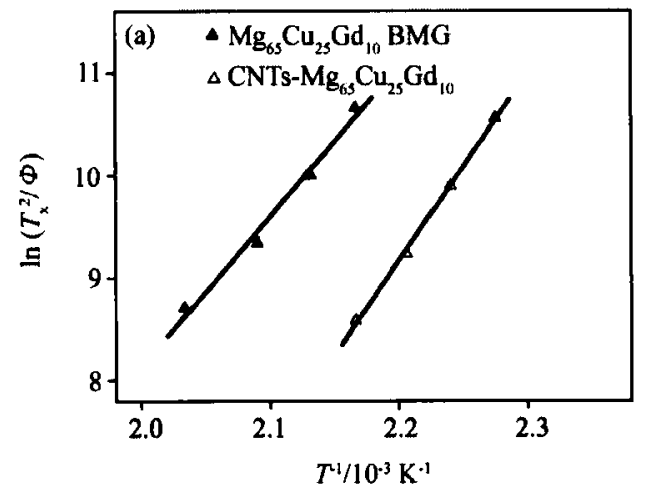

ter the addition of $\mathrm{CNTs}$, implying that the addition of CNTs degrades glass-forming ability of BMG matrix.

In the process of preparing BMG and its CNTs composite, cooling rate during quenching and sample size are kept as the same. The rate constant of crystalline reaction reflects GFA to some extent ${ }^{[16]}$. The relation between rate constant of crystalline reaction ane the temperature obeys Arrehenius law:

$\nu_{\mathrm{T}}=\nu_{0} \exp \left(-E / k_{\mathrm{B}} T\right)$

where $\nu_{0}$ is frequency factor. According to Kissinger Eq. (2), $\nu_{0}$ can be obtained from the slope and intercept of Kissinger plots. The calculated $\nu_{T}$ of BMG and its composite at crystallization peak are 0.333 and $0.522 \mathrm{~s}^{-1}$, respectively. The addition of CNTs increases the rate constant of crystalline reaction, and subsequently decreases GFA of BMG matrix. The same phenomena were also reported for $\mathrm{Zr}$-based CNTs-BMG composite ${ }^{[11]}$. It is argued that the formation of $\mathrm{ZrC}$ at the interface between amorphous matrix and CNTs leads to the decrease of GFA. However, there is no element, which can react with the added CNTs for Mg-based BMGs. In order to reveal the mechanism of this GFA decrease for Mg-based CNTs reinforced composite, EDX analysis of the matrix is carried out which is shown in Fig. 6 . EDX results

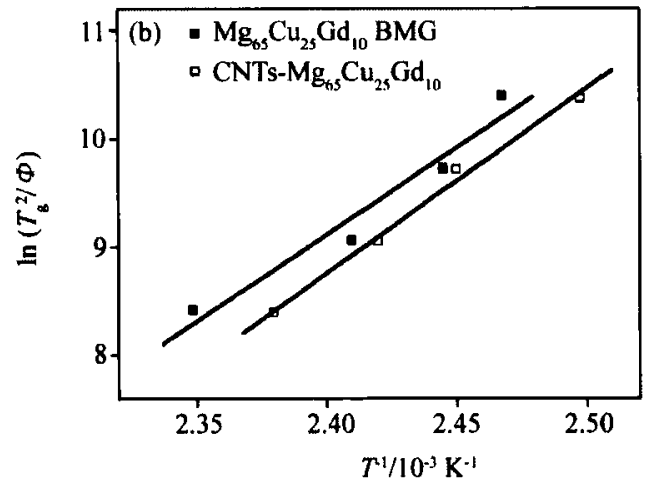

Fig.5 Kissinger plots of crystallization temperature (a) and glass transition (b) for Mg-Cu-Gd BMG and its CNTs composite
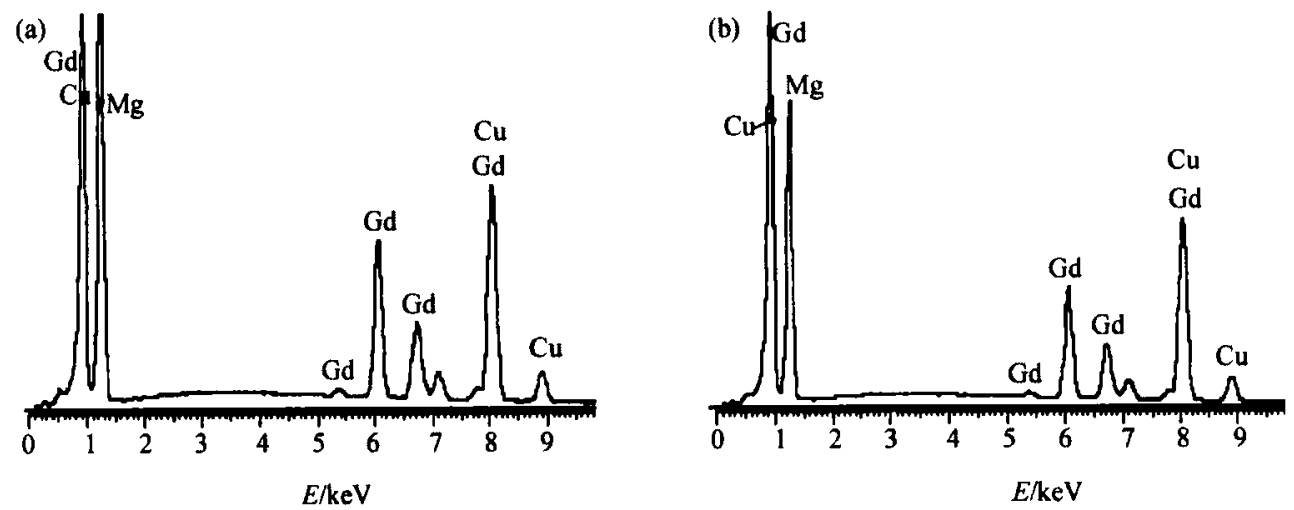

Fig.6 EDX spectra of Mg-Cu-Gd BMG and its CNTs composite (a) Mg-Cu-Gd BMG; (b) CNTs-BMG composite 
show that the compositions of undoped BMG and CNTs-BMG composite are $\mathrm{Mg}_{63.28} \mathrm{Cu}_{26.88} \mathrm{Gd}_{9.83}$ and $\mathrm{Mg}_{67.5} \mathrm{Cu}_{25.1} \mathrm{Gd}_{7.4}$, respectively. Considering the loss of $\mathrm{Gd}$ element is the same at the same cast condition, the relatively low Gd content in BMG composite should be induced by CNTs addition. Xi et al. ${ }^{[17]}$ studied the properties of $\mathrm{Mg}-\mathrm{Cu}-\mathrm{RE}$ ( $\mathrm{RE}$, rare earth metal) BMG. They found that GFA of BMG is very sensitive to $\mathrm{Gd}$ content, when the $\mathrm{Gd}$ atomic suffix is not equal to 10 , GFA of BMG decreases obviously and crystallization temperature shifts to lower temperature, which is very similar with our experimental results. Therefore, the decrease of GFA might be attributable to the change of $\mathrm{Gd}$ content in the matrix by CNTs addition .

\section{Conclusions}

1. Both the glass transition and crystallization process of Mg-based BMG and CNTs composite have kinetic effect. CNTs addition cannot change this feature. The relation between $T_{\mathrm{g}}, T_{\mathrm{x}}$ and heating rate can be well described by Lasoka equation. CNTs addition weakens the dependence of crystallization on heating rates.

2. The activation energy of glass transition of BMG as well as its CNTs composite was calculated by Kissinger equation. The activation energy increases after the addition of CNTs, indicating the decrease of glass-forming ability.

3. CNTs addition increases the rate constant of crystalline reaction, hence decreases GFA.

4. GFA decrease in CNTs composite is possibly attributable to the change of $\mathrm{Gd}$ content in the matrix induced by CNTs addition .

\section{References :}

[1] Inoue A. Stabilization of metallic supercooled liquid and bulk amorphous alloys $[\mathrm{J}]$. Acta Mater, 2000, 48 (1): 279 .

[2] Amiya K, Inoue A. Thermal stability and mechanical properties of $\mathrm{Mg}-\mathrm{Y}-\mathrm{Cu}-\mathrm{M}(\mathrm{M}=\mathrm{Ag}, \mathrm{Pd})$ bulk amorphous alloys [J]. Mater. Trans. JIM, 2000, 41(11): 1460.

[3] Yuan G, Zhang T, Inoue A. Thermal stability, glassforming ability and mechanical properties of $\mathrm{Mg}-\mathrm{Y}-\mathrm{Zn}-\mathrm{Cu}$ glassy alloys [J]. Mater. Trans., 2003, 44 (11): 2271.
[4] Men H, Kim D H J. Fabrication of ternary Mg-Cu-Gd bulk metallic glass with high glass-forming ability under air atmosphere $[\mathrm{J}]$. Mater. Res., 2003, 18(7): 1502.

[5] Han S M, Liu B Z, Zhang Z, et al. Phase structure and electrochemical properties of $\mathrm{RE}-\mathrm{Mg}$ based composite hydrogen storage alloys $[\mathrm{J}]$. Journal of Rare Earths, 2004, 22(6): 878 .

[6] Carneiro O, Covas J, Bernardo C, et al. Production and assessment of polycarbonate composites reinforced with vapour grown carbon fibres $[J]$. Co'mp. Sci. Technol., 1998, 58(3): 401 .

[7] Thostenson E T, Li W Z, Wang D Z, et al. Carbon nanotube/carbon fiber hybrid multiscale composites $[\mathrm{J}]$. J. Appl. Phys., 2002, 91(9): 6034 .

[8] Lau K T, Hui D. The revolutionary creation of new advanced materials-carbon nanotube composites $[\mathrm{J}]$. Composites Part B-Engineering, 2002, 33(4): 263.

[9] Thostenson E T, Ren Z F, Chou T W. Advances in the science and technology of carbon nanotubes and their composites: a review $[\mathbf{J}]$. Composites Science and Technology, 2001, 61(13): 1899.

[10] Harris P J F. Carbon nanotube composites [J]. International Materials Reviews, 2004, 49(1): 31 .

[11] Bian Z, Wang R J, Wang W H, et al. Carbon-nanotube-reinforced $\mathrm{Zr}$-based bulk metallic glass composites and their properties $[\mathrm{J}]$. Adv. Fuct. Mater., 2004, 14 (1): 55 .

[12] Zhuang Y, Zhao D, Zhang Y, et al. Kinetics of glass transition and crystallization in multicomponent bulk amorphous alloys $[J]$. Science in China (Series A), $2000,43(3): 1195$.

[13] Lasocka $M$. The effect of scanning rate on glass transition temperature of splat-cooled $\mathrm{Te}_{8 \mathrm{~s}} \mathrm{Ge}_{15}[\mathrm{~J}]$. Mater. Sci. Eng., 1976, 23(1): 173.

[14] Zhuang Yuanxin, Zhao Deqian, Zhang Yong, et al. Kinetics of glass transition and crystallization in multicomponent bulk amorphous alloys $[\mathrm{J}]$. Science in China (Series A), 2000, 43(11): 1195.

[15] Kissinger H E. Variation of peak temperature with heating rate in differential thermal analysis $[\mathrm{J}]$. J. Res . Nat. Bur. St., 1956, 57(1): 217.

[16] Xu H, Tan X H, Dong Y D. Crystalline behavior and magnetic properties of $\mathrm{Nd}_{60} \mathrm{Fe}_{30-x} \mathrm{Al}_{10} \mathrm{Co}_{x}(x=0,5,10)$ bulk amorphous alloys $[\mathrm{J}]$. Journal of Rare Earths, $2003,21(5)$ : 552 .

[17] Xi X K, Wang R J, Zhao D Q, et al. Glass-forming $\mathrm{Mg}-\mathrm{Cu}-\mathrm{RE}$ ( $\mathrm{RE}=\mathrm{Gd}, \mathrm{Pr}, \mathrm{Nd}, \mathrm{Tb}, \mathrm{Y}$, and Dy) alloys with strong oxygen resistance in manufacturability $[\mathrm{J}]$. J. Non-Crystalline Solids, 2004, 344(1) : 105. 